\title{
Japanese encephalitis vaccine-facilitated dengue virus infection-enhancement antibody in adults
}

\author{
Yuka Saito ${ }^{1,2}$, Meng Ling Moi ${ }^{3 *}$, Nozomi Takeshita ${ }^{4}$, Chang-Kweng Lim ${ }^{1}$, Hajime Shiba ${ }^{2}$, Kuniaki Hosono ${ }^{2}$, \\ Masayuki Saijo ${ }^{1}$, Ichiro Kurane ${ }^{5}$ and Tomohiko Takasaki ${ }^{6}$
}

\begin{abstract}
Background: Dengue virus (DENV) and Japanese encephalitis virus (JEV) belong to the genus Flavivirus, and infection with a virus within this genus induces antibodies that are cross-reactive to other flaviviruses. Particularly in DENV infection, antibodies to DENV possess two competing activities: neutralizing activity and infection-enhancing activity. These antibody activities are considered central in modulating clinical outcomes of DENV infection. Here, we determined the neutralizing and infection-enhancing activity of DENV cross-reactive antibodies in adults before and after JE vaccination.

Methods: Participants were 77 Japanese adults who had received a single dose of inactivated Vero cell-derived JE vaccine. A total of 154 serum samples were obtained either before or approximately a month after a single dose of JE vaccination. The antibody-dependent enhancement (ADE) activity to each of four DENV serotypes and the neutralizing activities to DENV and to JEV were determined in each of the serum samples by using baby hamster kidney (BHK) cells and FcyR-expressing BHK cells.

Results: A total of 18 post-JE immunization samples demonstrated cross-reactivity to DENV in an anti-DENV IgG ELISA. DENV neutralizing antibodies were not detected after JE vaccination in this study. However, undiluted post-JE vaccination serum samples from 26 participants demonstrated monotypic and heterotypic ADE activity to DENV. ADE activity was also observed in 1:10-diluted samples from 35 of the JE vaccine recipients $(35 / 77,45 \%)$.

Conclusion: In summary, JE vaccination induced DENV cross-reactive antibodies, and at sub-neutralizing levels, these DENV cross-reactive antibodies possess DENV infection-enhancement activity. The results also indicate that cross-reactivity to DENV is associated with high levels of JEV neutralizing antibodies and, the DENV cross-reactivity is further facilitated by JE vaccination.
\end{abstract}

\section{Background}

Dengue virus (DENV) infection mainly occurs in tropical and subtropical areas. It is estimated that there are 390 million dengue infections annually, and recently the number of dengue cases has increased exponentially worldwide [1]. Although DENV had not previously been endemic in Japan, a dengue outbreak occurred in 2014, resulting in 162 autochthonous dengue cases [2]. DENV and Japanese encephalitis virus (JEV) co-circulate in many Asian countries [3]. JE occurs in East, South, and

\footnotetext{
* Correspondence: sherry@nagasaki-u.ac.jp

${ }^{3}$ Department of Virology, Institute of Tropical Medicine, Nagasaki University,

Sakamoto 1-12-4, Nagasaki, Nagasaki 852-8523, Japan

Full list of author information is available at the end of the article
}

Southeast Asia. Both DENV and JEV cause serious public health threats and are the leading causes of hospitalization and death in children in vast regions of Asia. These viruses belong to the genus Flavivirus, and infection with a virus within this genus induces antibodies that are cross-reactive to other flaviviruses [4-6]. Particularly in DENV infection, antibodies induced has been demonstrated to possess 2 competing activities: neutralizing and infection-enhancement activities. Antibodies with DENV infection-enhancement activities are speculated to contribute to disease severity by a mechanism known as antibody-dependent enhancement (ADE). This mechanism leads to high levels of infection and the production of progeny viruses in DENV target 
cells, the FcyR-expressing monocytes and macrophages. Infection-enhancement activity also hampers virus neutralizing activity $[7,8]$.

DENV and JEV are antigenically related viruses. Crossreactivity was observed in vitro between both of these viruses $[4,5]$. An improved understanding of preexisting immunity to JEV and the implications of this flavivirus-primed immunity in subsequent infection are important as DENV outbreaks often occur in JEV coendemic areas as well as in areas where JE immunization is routine. Vaccination also induces cross-protection between JEV and viruses from the same Japanese encephalitis serocomplex-group including West Nile virus (WNV), and Murray Valley encephalitis virus in animal models $[9,10]$. Natural DENV and JEV infection or JE vaccine immunization, could each contribute to the overall immune background of an individual in areas where both DENV and JEV co-circulate $[11,12]$. Therefore, it is particularly challenging to define the association between pre-existing immunity to JEV and dengue clinical severity in DENV endemic areas. Although cross-protection against DENV was observed in mice inoculated with JE vaccine, previous investigators have also demonstrated that flavivirus antisera enhance DENV-2 infection [13]. These antibodies with neutralizing activity (NA) against JEV induced by either a prior JEV infection or by JE vaccination also possess neutralizing activity to DENV in vitro $[5,14]$. However, other investigators reported absence of cross-protective neutralizing antibodies of viruses from the same serocomplex group (JEV and WNV) $[15,16]$. Pre-existing immunity to JEV was also associated with an increased occurrence of symptomatic DENV infection [12]. Because non-Fc $\gamma R$ bearing cells were used in these studies, the sum of neutralizing activity and infection-enhancement activity, and the ADE activity of these antibodies at subneutralizing levels, has not been determined. Thus, a better understanding on cross-reactive immunity induced with JE vaccination, particularly the infection-enhancement and neutralizing activities of the cross-reactive antibodies, is expected to facilitate development of effective vaccination and preventive strategies for flavivirus disease control, including DENV and JEV infection [17-19].

Potential interactions between flaviviruses have significant public health implications because areas with high JE vaccine coverage and JEV circulation are also frequently areas with a high dengue disease burden [12]. With the rapid expansion of DENV endemic areas and emergence in previously non-endemic areas, an improved understanding of the pre-existing immunity against JEV and of the potential implications for subsequent DENV exposure is necessary. In this study, we used BHK and FcyR-expressing BHK cells to determine DENV cross-reactive activities in antibodies of adults with pre-existing immunity to JEV, and those facilitated by JE vaccination.

\section{Methods}

\section{Serum samples}

Serum samples were obtained from 77 Japanese adults $($ mean age $=38.1 \pm 10.7$; male:female ratio $=1.8: 1)$ who received a single dose of Vero cell-derived inactivated JE vaccine (JEBIK V ${ }^{\circ}$, BIKEN, Japan) at the National Center for Global Health and Medicine (NCGM), Tokyo Japan [20]. These serum samples were obtained before vaccination and 3-5 weeks after JE vaccination during the period of 2009-2011 (Additional file 1 Table S1), during which no local DENV outbreaks were reported [20]. Samples were collected at the NCGM, and all laboratory tests were performed at the National Institute of Infectious Diseases, Japan (NIID). The study protocols were approved by the NCGM and NIID Institutional Ethics Review Board (no. 473). All samples were de-identified prior to tests conducted at the NIID. All serum samples were heat-inactivated at $56{ }^{\circ} \mathrm{C}$ for $30 \mathrm{~min}$ prior to use. Of the 79 participants who received the JE vaccine, 50 participants had previous immunization history against JE, however the JE vaccination history for the other 29 participants was unknown. Samples from 2 participants were excluded from this study because of insufficient sample volume. Thus, samples from 77 participants were used in this study. Because the travel history of the participants was incomplete, that data were excluded from this study.

\section{Determination of the levels of anti-DENV IgM and IgG antibodies by ELISA}

The amount of DENV-specific IgM antibody in the serum samples was determined using an IgM capture ELISA kit (Dengue Virus IgM Capture DxSelect ELISA Kits, Focus Diagnostics, CA, USA) according to the manufacturer's instructions. Dengue IgG ELISAs (Dengue IgG Indirect ELISA, Panbio, Queensland, Australia and Dengue ELISA IgG, Vircell, Granada, Spain) were used according to manufacturer's instructions for the detection of anti-DENV IgG antibody. The presence of DENV cross-reactive IgG antibody was defined as positive detection by at least one of these kits [21]. The index value of anti-DENV IgM and IgG ELISA was determined by dividing the average OD of each sample by the cut-off value according to manufacturers' instruction. The cut-off value was determined by multiplying the average OD of the calibrator by the calibration factor provided by the manufacturer. Index values of $<0.9$, 0.9-1.1, and, >1.1 were considered negative, equivocal, and positive, respectively. Equivocals were regarded as negative. 


\section{Determination of the levels of anti-JEV IgG antibody by ELISA}

Anti-JEV IgG antibody in samples was determined by using an in-house JEV-specific IgG ELISA. A 96-well ELISA plate (NUNC, Thermo Scientific, USA) coated overnight at $4{ }^{\circ} \mathrm{C}$ with JEV antigen was used. After washing four times with $1 \times$ Dulbecco's phosphate buffered saline, no calcium, no magnesium ( $1 \times$ DPBS (-)) (Life Technologies, CA, USA), and $200 \mu \mathrm{l}$ of $0.1 \%$ casein in $1 \times$ DPBS (-) was added into each well, and the plate was incubated at $37^{\circ} \mathrm{C}$ for $2 \mathrm{~h}$. After washing, $100 \mu \mathrm{l}$ of the ten-folds diluted serum samples $(1: 100$ to $1: 25,600)$ were added and the plates were incubated at $37{ }^{\circ} \mathrm{C}$ for $1 \mathrm{~h}$. After washing, $100 \mu \mathrm{l}$ of horseradish peroxidase (HRP)-conjugated anti-human IgG was then added into each well, and the plate was incubated at $37{ }^{\circ} \mathrm{C}$ for $1 \mathrm{~h}$. After further washing, $100 \mu \mathrm{l}$ of tetramethylbenzidine (TMB) solution was added and reactions were terminated after $10 \mathrm{~min}$ with $100 \mu \mathrm{l}$ of stop solution $(1 \mathrm{~N}$ $\mathrm{H}_{2} \mathrm{SO}_{4}$ ). The ELISA endpoint corresponded to the highest dilution of the serum sample in which the positive to negative $(\mathrm{P} / \mathrm{N})$ ratio of the O.D. values was greater than 2. Thus, samples of $\mathrm{P} / \mathrm{N}$ ratio of $>2$ were interpreted as positive.

\section{Viruses and cell lines}

DENV-1 (01-44 strain), DENV-2 (TLC-30 strain), DENV-3 (NRT 09-34 strain), and DENV-4 (08-11 strain) were used in neutralizing and ADE assays against DENV [22]. JEV (Beijing-1 strain) was used in the JEV neutralizing assays. Baby hamster kidney (BHK) cells (Japan Health Science Research Resources Bank, Japan), FcyR-expressing BHK cells and Vero cells (African green monkey kidney epithelial cells) were used [21, 22]. BHK cells and Vero cells were cultured in Eagle's Minimum Essential Medium (EMEM, Sigma), supplemented with heat-inactivated $10 \%$ fetal bovine serum (FBS, Sigma) without antibiotics. The cells were maintained at $37{ }^{\circ} \mathrm{C}$ in $5 \% \mathrm{CO}_{2}$. FcyR-expressing BHK cells were cultured in EMEM, supplemented with heat-inactivated $10 \%$ FBS and $0.5 \mathrm{mg} / \mathrm{ml}$ neomycin (G418, Roche Diagnostics GmbH Mannheim, Germany) at $37{ }^{\circ} \mathrm{C}$ in $5 \% \mathrm{CO}_{2}$.

\section{JEV and DENV neutralization assay}

Neutralization tests against JEV were performed by using the $50 \%$ focus reduction neutralization test method $\left(\mathrm{FRNT}_{50}\right)$ as previously reported [20]. Neutralization tests against DENV were performed by the $50 \%$ plaque reduction neutralization test $\left(\mathrm{PRNT}_{50}\right)$ method as previously reported [22]. The presence of a NA titer was defined as the demonstration of a $50 \%$ or greater plaque reduction at a titer of $\geq 1: 10$. The values of neutralizing antibody titers of below limit of detection $(<1: 10)$ were considered equal to the limit of detection divided by the square root of 2 .
Antibody-dependent enhancement (ADE) assay for DENV The presence of ADE activity was determined by plaque assay. A total of 154 serum samples was diluted to 1:10 in EMEM supplemented with 10 \% FBS. DENV were prepared at titers of $2000 \mathrm{PFU} / \mathrm{ml}$ for ADE assays using 10-fold-diluted serum samples. Virus-antibody mixtures were prepared by mixing $25 \mu \mathrm{l}$ each of each DENV serotype with $25 \mu \mathrm{l}$ of diluted serum samples. A control mixture was prepared by combining $25 \mu$ l of each DENV serotype with $25 \mu \mathrm{l}$ of EMEM supplemented with $10 \%$ FBS. For samples that demonstrated ADE activity at a 1:10 dilution, further tests was performed using undiluted serum samples [23]. Infection enhancement (measured as ADE activity) was tested using serum samples that had been diluted 1:10. ADE activity was defined as fold-enhancement values greater than the cut-off value (cut-off value = mean plaque count FcyR-expressing BHK cells without the addition of human serum using +3 s.d.)/(mean plaque count of Fc $\gamma$ R-expressing BHK cells without the addition of human serum +3 ). The presence of ADE activity was defined as having fold enhancement values of $>1.9$ for DENV-1, DENV-2, and DENV-3 or of $>1.8$ for DENV-4.

\section{Statistical analyses}

The tabulation, management, and analyses of data were performed using Microsoft Excel. Study outcomes were compared by $t$-tests, chi-squared tests, Fisher's exact tests and Pearson's correlation test [24, 25]. Geometric datasets were used for parametric analyses.

\section{Results}

Increase in positive rates of anti-DENV IgG after JE vaccination

The overall serological cross-reactivity to DENV in adult JE vaccine recipients was examined by anti-DENV IgG ELISAs. ELISA was performed using 154 serum samples obtained from participants pre- and post-JE vaccination (Additional file 1: Table S1). Prior to JE vaccination, seropositive rate of DENV IgG seropositivity was $9 \%(3 / 34)$ in samples with no JE neutralizing antibodies, and was $21 \%$ (9/43) in samples with JEV neutralizing antibodies. After JE vaccination, anti-DENV IgG antibodies was detected in $20 \%(1 / 5)$ samples without JEV neutralizing activity (PRNT50<1:10), and in $40 \%(29 / 72)$ samples with JEV neutralizing activity (Fig. 1a). Overall, the number of participants that demonstrated DENV cross-reactive IgG antibody increased from 12 to 30 after JE vaccination. DENV reactivity was defined as positive if a sample was determined to be positive by either of the two ELISA platforms used (Additional file 2: Table S2). The anti-DENV IgG index values were higher after JE vaccination $(t$-test $p<0.01)$ (Fig. 1b-c). Thus, the results indicate that the increase 


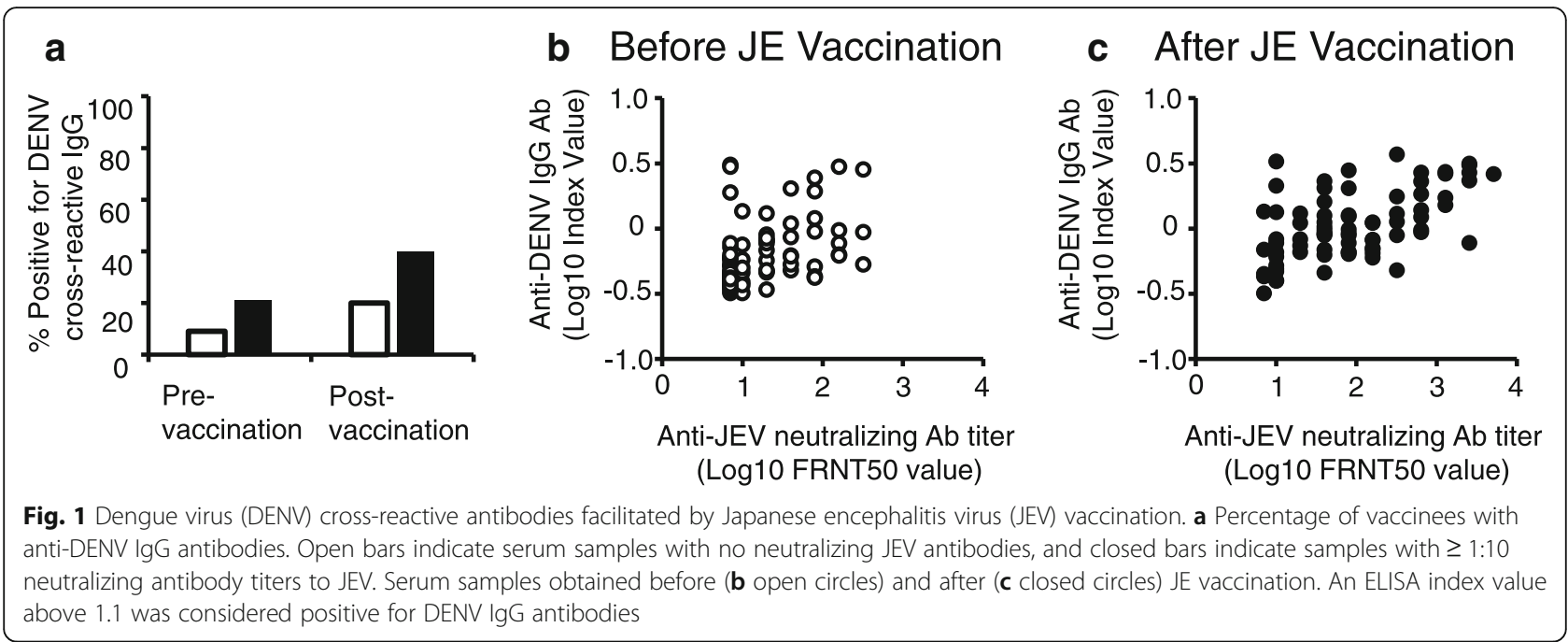

in DENV cross-reactive IgG antibodies after JE vaccination moderately correlated with the levels of JEV neutralizing activity.

\section{JE vaccination increased ADE activity to all four DENV serotypes}

Vacinee serum samples at 1:10 dilutions were examined for ADE activity against each of the four DENV serotypes (DENV 1-4) by using Fc $\mathrm{R}$-expressing BHK cells. The ADE activity against DENV-1 was $6 \%(5 / 77)$ before vaccination and $18 \%(14 / 77)$ after vaccination. For DENV2, $3 \%(2 / 77)$ had ADE activity before JE vaccination and $21 \%(16 / 77)$ after vaccination, for DENV3, $14 \%(11 / 77)$ had ADE activity before vaccination and $31 \%(24 / 77)$ after vaccination, and for DENV4, $13 \%(10 / 77)$ had ADE activity before vaccination and $34 \%(26 / 77)$ after vaccination. The number of serum samples observed with ADE activity to each of the four DENV serotypes significantly increased after JE vaccination (Fisher's exact test): DENV$1(p=0.03), \mathrm{DENV}-2(p<0.01), \mathrm{DENV}-3(p<0.01)$, and DENV-4 $(p<0.01)$ (Table 1$)$. The results indicate that cross-reactive activity to DENV increase with JE vaccination and these activities possess ADE activity to DENV.

\section{Increase in overall ADE activity to DENV in JE vaccine} recipients

The ADE activity among the four DENV serotypes and the levels of JEV neutralizing activity before and after vaccination were also examined. Serum samples were split into three groups: Group A, JE vaccine recipients that did not possess ADE activity against DENV before or after JE vaccination; Group B, JE vaccine recipients that exhibited ADE activity to DENV only after JE vaccination; and Group C, JE vaccine recipients that exhibited ADE activity to DENV both before and after JE vaccination. JEV NA titers of subjects in Group A were significantly lower before and after vaccination than those of subjects who demonstrated ADE activity to DENV after JE vaccination (Group B and Group C). The increase in JEV neutralizing activity levels in participants with ADE activity to DENV after JE vaccination was significantly higher in participants that demonstrated ADE after vaccination, in comparison to the participants that did not exhibit ADE activity to DENV (Group B $=578 \pm$ 803, $t$-test $p<0.01$; Group $C=980 \pm 1422, t$-test $p<$ 0.01 ). Additionally, the number of participants that demonstrated DENV IgG cross-reactive antibodies increased in all groups (Groups A-C). Specifically, the anti-DENV

Table 1 Number of serum samples with antibody-dependent enhancement (ADE) activity ${ }^{a}$ to each of the 4 dengue virus (DENV) serotypes, pre- and post-Japanese encephalitis (JE) vaccination

\begin{tabular}{lllll}
\hline Samples & \multicolumn{4}{l}{ Number of serum samples that demonstrated ADE activity (Percentage of samples with ADE activity) } \\
\cline { 2 - 5 } & DENV-1 & DENV-2 & DENV-3 & DENV-4 \\
\hline $\begin{array}{l}\text { Pre-JE vaccination } \\
(n=77)\end{array}$ & $5(6 \%)$ & $2(3 \%)$ & $11 \%(13 \%)$ \\
$\begin{array}{l}\text { Post-JE vaccination } \\
(n=77)\end{array}$ & $14(18 \%)$ & $16(21 \%)$ & $24(31 \%)$ & $24(31 \%)$
\end{tabular}

${ }^{a}$ The number of serum samples observed with ADE activity to each of the four DENV serotypes significantly increased after JE vaccination (Fisher's exact test): DENV-1 $(p=0.03)$, DENV-2 $(p<0.01)$, DENV-3 $(p<0.01)$, and DENV-4 $(p<0.01)$ 
IgG levels increased significantly after JE vaccination in Group B as compared to those in Group A (Group $\mathrm{A}_{\mathrm{POST}}=0.8 \pm 0.3$, Group $\mathrm{B}_{\mathrm{POST}}=1.5 \pm 0.7 ; t$-test $p<$ 0.01 ), with Group B demonstrating 15 DENV seroconversions after JE vaccination (Table 2).

Notably, the JE vaccine recipients in Group C demonstrated significantly higher anti-DENV IgG levels both pre- $(t$-test; $p<0.01$ against Groups $\mathrm{A}$ and $\mathrm{B})$ and post-JE vaccination ( $t$-test; $p<0.01$ against Groups A and B) than those in groups A and B. Group $\mathrm{C}$ also demonstrated a higher percentage of JE vaccine recipients with $\mathrm{ADE}$ activity against two or more DENV serotypes $(73 \%, 11 / 15)$, as compared to Group B $(50 \%, 10 / 20)$. The results, together with the observed higher JEV neutralizing activity in serum samples from Group C, suggested that pre-existing JEV immunity increased the overall immune reactivity against JEV and DENV due to facilitation by JE vaccination (Table 2).

Of the 23 participants who demonstrated anti-IgG DENV cross-reactive antibodies after JE vaccination, all had serum samples that were negative for anti-DENV IgM (data not shown), indicating that the increase in anti-DENV IgG levels after JE vaccination was not due to a recent DENV infection.

\section{$A D E$ activity to DENV in undiluted serum samples after JE vaccination}

The ADE activity of the serum samples with demonstrated ADE activity against DENV at 1:10 dilutions after JE vaccination were also used to determine the
ADE activity in undiluted samples on FcyR-expressing BHK cells. Out of the 35 serum samples that demonstrated ADE activity to DENV at a 1:10 dilution, 26 undiluted serum samples also demonstrated ADE activity to DENV. The fold-enhancement of undiluted serum samples ranged from 2.5 -fold to 14.5 -fold. Of these 26 serum samples, 10 serum samples demonstrated ADE activity against a single serotype, 4 serum samples demonstrated ADE activity against two DENV serotypes, and 12 serum samples demonstrated ADE activity against three or more DENV serotypes, at undiluted levels (Table 3). Serum dilution decreases the amount of antibodies, and thus, some samples exhibited ADE activity upon serum dilution. Using undiluted serum samples, the results indicated that these serum samples possessed the potential to enhance DENV infection at biological levels.

\section{Absence of NA to DENV in serum samples collected from $J E$ vaccine recipients}

The DENV neutralizing activity against all four serotypes of the serum samples obtained from 77 JE vaccine recipients was determined using BHK and FcyR-expressing BHK cells. Out of the $77 \mathrm{JE}$ vaccine recipients, only one (vaccine recipient \#73) exhibited neutralizing activity against DENV-1 in the serum samples collected both before and after JE vaccination (BHK cells, PRNT $_{50}$ DENV1 $1_{\text {PRE }}=160, \quad$ PRNT $_{50} \quad$ DENV $1_{\text {POST }}=160 ; \quad$ Fc $\gamma R-$ expressing BHK cells, $\mathrm{PRNT}_{50} \mathrm{DENV}_{\mathrm{PRE}}=40$, PRNT50 DENV1 $\left.1_{\text {POST }}=40\right)$. Subject $\# 73$ also exhibited ADE to DENV-1, pre- and post-JE vaccination, from serum

Table 2 Antibody-dependent enhancement (ADE) activity against the four dengue virus (DENV) serotypes in each of the samples obtained from the 77 participants, pre- and post-Japanese encephalitis (JE) vaccination and corresponding JEV neutralizing antibody (NA) levels

\begin{tabular}{|c|c|c|c|}
\hline \multirow[t]{2}{*}{ ADE activity in each of the serum samples to four DENV serotypes } & \multirow[t]{2}{*}{$\mathrm{n}$} & \multicolumn{2}{|c|}{$\begin{array}{c}\text { JEV NA titer } \\
\text { (Mean JEV NA titer } \pm \text { s.d.) }\end{array}$} \\
\hline & & Pre-vaccination & Post-vaccination \\
\hline (A) Absence of ADE activity to DENV, both before and after JE vaccination & 40 & $19 \pm 29$ & $153 \pm 419$ \\
\hline \multicolumn{4}{|l|}{ (B) Presence of ADE activity to DENV after JE vaccination only } \\
\hline 1. ADE activity to $\geq 3$ DENV serotypes & 6 & $98 \pm 124$ & $1130 \pm 1199$ \\
\hline 2. ADE activity to two DENV serotypes & 4 & $91 \pm 153$ & $500 \pm 592$ \\
\hline 3. ADE activity to one DENV serotype & 10 & $47 \pm 64$ & $277 \pm 372$ \\
\hline Total & 20 & $71 \pm 101^{b}$ & $578 \pm 803^{b}$ \\
\hline \multicolumn{4}{|l|}{ (C) Presence of ADE activity to DENV both before and after JE vaccination } \\
\hline 1. ADE activity to $\geq 3$ DENV serotypes & 10 & $96 \pm 122$ & $1325 \pm 1644$ \\
\hline 2. ADE activity to two DENV serotypes & 1 & 40 & 80 \\
\hline 3. ADE activity to one DENV serotype & 4 & $53 \pm 74$ & $341+346$ \\
\hline Total & 15 & $81 \pm 106^{\mathrm{b}}$ & $980+1422^{b}$ \\
\hline
\end{tabular}

aJEV NA titers were determined by using a $50 \%$-focus-reduction neutralization test

${ }^{b}$ The increase in JEV NA levels in participants with ADE activity to DENV after JE vaccination was significantly higher in participants that demonstrated ADE after vaccination (Group $B=578 \pm 803$, $t$-test $p<0.01$; Group $C=980 \pm 1422$, $t$-test $p<0.01$ ) in comparison to those that did not demonstrate ADE activity to DENV (Group A). Samples from 2 patients were excluded due to insufficient volume for the ADE assay 
Table 3 Antibody-dependent enhancement (ADE) activity against four dengue virus (DENV) serotypes in post-Japanese encephalitis (JE) vaccination serum samples that were diluted 1:10 or left undiluted

\begin{tabular}{|c|c|c|c|c|c|c|c|c|c|c|}
\hline \multirow{3}{*}{$\begin{array}{l}\text { Sample no. } \\
\text { (post-JE vaccination) } \\
(n=35)^{c}\end{array}$} & \multicolumn{8}{|c|}{ Fold enhancement to DENV ${ }^{a}$} & \multirow{2}{*}{\multicolumn{2}{|c|}{$\begin{array}{l}\text { ADE activity to } \\
\text { number of DENV } \\
\text { serotypes }\end{array}$}} \\
\hline & \multicolumn{2}{|c|}{ DENV-1 } & \multicolumn{2}{|l|}{ DENV-2 } & \multicolumn{2}{|l|}{ DENV-3 } & \multicolumn{2}{|l|}{ DENV-4 } & & \\
\hline & Undiluted & 1:10-dilution & Undiluted & 1:10-dilution & Undiluted & 1:10-dilution & Undiluted & 1:10-dilution & Undiluted & 1:10-dilution \\
\hline 3 & $-{ }^{\mathrm{b}}$ & - & - & - & - & - & 3.1 & 2.5 & 1 & 1 \\
\hline 9 & - & - & - & - & - & - & 2.2 & 1.9 & 0 & 1 \\
\hline 11 & $4.5^{\mathrm{a}}$ & 2.3 & 3.2 & 4.0 & $4.1^{\mathrm{a}}$ & 2.9 & 2.3 & 2.7 & 3 & 4 \\
\hline 13 & 13.0 & 2.8 & 4.0 & 2.8 & 5.9 & 2.1 & 4.5 & 2.8 & 4 & 3 \\
\hline 19 & - & - & - & - & 4.4 & 2.6 & - & - & 1 & 1 \\
\hline $20^{2}$ & 14.5 & 2.6 & 2.8 & 3.6 & 5.3 & 2.5 & 3.3 & 2.4 & 4 & 4 \\
\hline $21^{1}$ & - & - & 4.2 & 2.6 & 4.3 & 3.5 & 4.9 & 2.8 & 3 & 3 \\
\hline 22 & 3.5 & 2.0 & - & - & - & - & - & - & 1 & 1 \\
\hline $31^{3}$ & 2.5 & 3.0 & 6.6 & 4.8 & 2.7 & 3.2 & 4.7 & 2.4 & 2 & 4 \\
\hline $32^{1}$ & - & - & - & - & 2.5 & 2.5 & - & - & 0 & 1 \\
\hline 33 & 6.0 & 2.5 & 2.5 & 3.8 & 4.1 & 3.9 & 4.4 & 2.2 & 4 & 4 \\
\hline 43 & - & - & 5.1 & 2.8 & 4.9 & 4.8 & 6.6 & 1.8 & 3 & 2 \\
\hline 47 & - & - & - & - & 3.2 & 3.3 & 1.6 & 2.3 & 0 & 2 \\
\hline 49 & - & - & - & - & 3.9 & 2.3 & - & - & 1 & 1 \\
\hline $51^{2}$ & 6.7 & 2.6 & - & - & 5.0 & 2.6 & 3.3 & 2.3 & 3 & 3 \\
\hline 53 & - & - & - & - & 3.9 & 2.0 & - & - & 1 & 1 \\
\hline 54 & - & - & - & - & 5.0 & 3.5 & - & - & 1 & 1 \\
\hline $57^{3}$ & 6.7 & 2.2 & 5.5 & 2.0 & & & 4.5 & 2.7 & 3 & 3 \\
\hline 58 & 4.0 & 1.9 & 1.5 & 3.2 & 4.1 & 3.2 & 3.4 & 3.8 & 3 & 3 \\
\hline $59^{3}$ & 4.3 & 4.2 & 1.2 & 2.5 & 4.0 & 3.9 & 1.0 & 3.4 & 2 & 4 \\
\hline 61 & 7.3 & 2.1 & - & - & - & - & - & - & 1 & 1 \\
\hline $73^{2}$ & - & - & 0.4 & 2.3 & 4.1 & 3.5 & 1.3 & 3.5 & 1 & 3 \\
\hline 77 & - & - & - & - & 6.3 & 4.0 & 3.3 & 1.8 & 2 & 1 \\
\hline $78^{1}$ & - & - & - & - & 2.0 & 2.2 & - & - & 0 & 1 \\
\hline 79 & 2.0 & 2.2 & 1.1 & 3.6 & 5.0 & 6.7 & 3.8 & 2.7 & 2 & 4 \\
\hline 80 & 4.0 & 2.1 & 2.5 & 2.5 & 5.4 & 3.2 & - & - & 3 & 3 \\
\hline 85 & - & - & - & - & - & - & 6.2 & 2.6 & 1 & 1 \\
\hline $86^{1}$ & 1.7 & 2.4 & 1.0 & 3.3 & 2.9 & 4.6 & 2.3 & 2.5 & 0 & 4 \\
\hline $89^{3}$ & 7.0 & 4.1 & 4.2 & 2.0 & 4.8 & 5.1 & 3.3 & 3.3 & 4 & 4 \\
\hline
\end{tabular}


Table 3 Antibody-dependent enhancement (ADE) activity against four dengue virus (DENV) serotypes in post-Japanese encephalitis (JE) vaccination serum samples that were diluted 1:10 or left undiluted (Continued)

\begin{tabular}{|c|c|c|c|c|c|c|c|c|c|c|}
\hline 93 & - & - & - & - & 3.1 & 3.2 & 1.9 & 2.4 & 0 & 2 \\
\hline $94^{1}$ & - & - & - & - & - & - & 5.8 & 2.6 & 1 & 1 \\
\hline $95^{1}$ & - & - & - & - & - & - & 2.1 & 2.1 & 0 & 1 \\
\hline $96^{1}$ & - & - & 4.0 & 2.0 & 3.8 & 3.1 & 3.8 & 5.2 & 3 & 3 \\
\hline 100 & - & - & - & - & - & - & 2.4 & 2.1 & 0 & 1 \\
\hline 110 & - & - & - & - & 4.9 & 2.8 & 3.4 & 2.2 & 2 & 2 \\
\hline Total number of samples & 12 & 14 & 11 & 16 & 20 & 24 & 17 & 24 & & \\
\hline
\end{tabular}

attalics indicates the presence of ADE activity

b-denotes an absence of ADE activity at a 1:10 sample dilution to the tested DENV serotype. Assessments of ADE activity in undiluted samples were not performed in samples that did not demonstrate ADE activity at the $1: 10$ dilution

'The numbers in superscript correspond to the number of serotypes to which a sample had ADE activity prior to JE vaccination in this study 
dilutions of 1:160 to 1:2560. Pre- and post-JE vaccination fold-enhancement values to DENV1 of subject \#73 ranged from 2.0 to 5.9. The results suggest that participant \#73 was previously infected with DENV-1. With the exception of participant \#73, the absence of DENV neutralizing activity against all four DENV serotypes in our samples suggests that prior exposure to DENV was likely limited in the JE vaccine recipients of this study. Thus, although the ADE activity increased, DENV crossreactive NAs were overall absent in this study.

\section{Anti-JEV IgG endpoints were higher than anti-DENV IgG endpoints in samples that were positive for anti-DENV lgG}

Twelve of the serum samples collected prior to JE vaccination and positive for anti-DENV IgG antibodies were selected, and the anti-JEV IgG ELISA endpoints of these 12 serum samples were compared with their corresponding anti-DENV IgG ELISA endpoints (Additional file 3: Table S3). The JEV endpoints in all 12 samples were higher than those for DENV (mean JEV endpoint $=9600 \pm 9887$, mean DENV endpoint $=600 \pm 618$; $t$-test $p<0.01)$. The results demonstrated that in these serum samples, the levels of JEV antigen-reactive IgG were more than 10 times higher than those of DENV antigenreactive IgG. This suggested that the presence of DENV cross-reactive antibodies in these participants was likely facilitated by JE vaccination, rather than of natural DENV infection.

\section{Discussion}

We found that the increased occurrence of DENV crossreactive antibodies was most pronounced in individuals with higher levels of JEV neutralizing activity. Correspondingly, the number of participants with no crossreactive activity to DENV exhibited lower levels of JEV neutralizing activity. Other investigators have reported that cross-reactive antibodies induced by JE vaccination induced antibodies that neutralizes other flaviviruses including WNV and DENV [9, 14], while other groups have demonstrated the absence of neutralizing antibodies to other flaviviruses immunization against JE [15]. These studies however, used non-FcyR-expressing cells as assay cells. Since non-FcyR-expressing cells detects only neutralizing activity and not ADE, only cross-reactive neutralizing activity was reported [22]. Thus, the biological relevance of cross-reactive antibodies at subneutralizing levels was not determined. As antibodies induced at sub-neutralizing levels, efficient uptake of these virus-immune complex via the FcyRbearing cells can increase both the number of cells infected and the virus yield [26, 27]. ADE assays using FcyR-bearing cells have proved useful for functional studies of the receptor, and in the determination of ADE activity of human sera and for defining the role of antibodies in DENV pathogenesis [28, 29]. In our study, FcyR-expressing BHK cell lines detected both neutralizing activity and ADE activity. We demonstrated that flavivirus cross-reactive antibodies were induced after immunization, and these subneutralizing cross-reactive antibodies enhanced DENV infection. Given that neutralizing antibodies have been speculated to play a dominant role in defining infection outcomes during the early phase of virus entry, they may serve to block cell attachment of virus-immune complexes to a non-FcyR-bearing cell, or inhibit virus intracellular fusion after internalization [27, 30, 31]. The FcyR may also serve a regulatory role in viral entry, and intracellular fusion and virus production, by initiating cellular responses mediated by differential Fc-effector functions, which depends on the balance of neutralizing and ADE.

Undiluted serum samples may better reflect in vivo conditions than the use of diluted samples [23]. In this study, ADE activity against all four DENV serotypes was determined by using undiluted serum samples in this study. Of note, undiluted serum samples with high JEV neutralizing activity titers also exhibited ADE activity to DENV in undiluted serum samples (Table 3). Serum dilution dilutes the amount of total antibodies, resulting in lesser amount of cross-reactive antibody. Using convalescent serum samples from DENV patients, an ADE titration curve demonstrated: (1) virus neutralization at lower dilutions, followed by (2) ADE at higher dilutions, and finally (3) absence of cross-reactivity at even higher dilutions [23]. Serum samples with subneutralizing levels of antibodies exhibited patterns of (2) and (3), in the ADE titration curve. In this study, none of the samples demonstrated cross-reactive neutralizing antibody to DENV. Thus, in some undiluted serum samples that demonstrated ADE activity, further dilution of these samples could have further led to the decrease in, or even absence of cross-reactive antibodies [patterns (2) and (3)] (Table 3). Although the association between ADE levels and severity of DENV infection needs further clarification, previous studies have suggested that ADE in undiluted samples confers higher viremia, which is a prelude to severe DENV symptoms [32-34].

In mice, JE vaccination induced DENV cross-protective immunity and virus neutralizing activity [14]. Neutralizing activity against JEV induced by a prior JE vaccination was also demonstrated to possess neutralizing activity against DENV [14]. Studies have also shown that JE vaccines have limited effect on the severity of dengue disease [35]. However, there is limited and inconclusive evidence regarding pre-existing immunity to JEV and subsequent symptomatic or severe presentation of DENV infection [12, 36]. In flavivirus infection, the ability to mount an early and vigorous antibody response is associated with better clinical outcomes, indicating that anamnestic, anti-flavivirus immune response induced by prior DENV and JEV infection, 
for example, is important in conferring protection, or comparatively milder disease outcomes [37-39]. In this study, history of past JE and yellow fever (YF) vaccine immunization and travel history was not available. Thus, it was not possible to conclude the contributive effects of prior vaccination or possible exposure to flavivirus during travel on the background immunity. Related discrepancies due to background immunity could be attributed to the subsequent host response to immunization and, in turn, may have contributed to the induction of ADE activity to DENV after immunization against JE in some of the participants. Although there is an association with the magnitude of ADE activity and disease severity, the mechanisms of $\mathrm{ADE}$ in vivo remains elusive. Other underlying biological factors include cellular immunity, viral pathogenesis, and host genetic background, all of which are speculated to be involved in the pathogenesis of severe dengue. In this study, we used Fc $\gamma$ R-expressing BHK cells to determine the levels of ADE activity in the absence of other myeloid-specific receptors that may cooperatively facilitate infection and mediation of innate immunity. Presence of ADE antibodies may be one of the contributing factors to disease severity, however, subsequent studies using $\mathrm{F} c \gamma \mathrm{R}$ and other myeloid-specific receptors would better define the mechanisms of these antibodies in ADEmediated immunopathology.

The JE vaccine recipients in this study may be generally characterized as having no prior DENV exposure (i.e., samples collected from a non-DENV endemic area with an absence of DENV NA). Given that JEV continues to co-circulate with DENV in endemic areas, samples in such areas may demonstrate immunity to DENV which is facilitated by either DENV or JEV infection. In this study, the effect of JE vaccination was determined using pre- and post-JE vaccination samples obtained from a non-DENV endemic area. The possibility of recent DENV infection was excluded because all vaccinees were negative for anti-DENV IgM antibodies, and the anti-JEV IgG titers were comparatively higher in these vaccinees as compared to the levels of anti-DENV IgG (Additional file 3: Table S3). Thus, the results indicate that the DENV crossimmunity in this study was induced by JE vaccination. Taken together, our results support the hypothesis that DENV cross-reactive immunity facilitated by JE vaccination may plays two competing roles: (1) at sub-neutralizing levels, these antibodies enhances DENV infection, potentially leading to symptomatic infection and severe disease presentation, and (2) JE vaccination elicits a flavivirus amnestic response that is important for disease protection, which may result in milder disease outcomes. As ADE may reflect some aspects of DENV infection in vivo, our results suggest the necessity of further studies on the longterm impact of flavivirus vaccination on DENV clinical outcomes.

\section{Conclusions}

In summary, JE vaccination induced DENV crossreactive antibodies, and at sub-neutralizing levels, these antibodies demonstrated ADE to DENV. Additionally, our study highlighted that cross-reactivity to DENV is associated with high levels of JEV NAs and the DENV cross-reactivity is further facilitated by JE vaccination. Our study suggests that the immunological background and interplay between JEV and DENV influences the landscape of heterologous flavivirus immunity, with JE vaccination facilitating and setting the critical threshold of ADE and neutralizing activities of DENV crossreactive antibodies.

\section{Additional files}

Additional file 1: Table S1. JEV NA in 77 Japanese adults pre- and post-JE vaccination. (DOC $35 \mathrm{~kb}$ )

Additional file 2: Table S2. Detection rates of anti-DENV IgG antibody using Panbio and Vircell kits. (DOC 28 kb)

Additional file 3: Table S3. Anti-JEV and anti-DENV lgG endpoint titers after JEV vaccination as determined by ELISA. (DOC $35 \mathrm{~kb}$ )

\section{Abbreviations \\ ADE: Antibody-dependent enhancement; BHK: Baby hamster kidney; DENV: Dengue virus; JEV: Japanese encephalitis virus; NA: Neutralizing antibody; WNV: West Nile virus}

\section{Acknowledgements}

We thank Dr. Jeffrey V. Ravetch (Rockfeller University, NY, USA) for generously providing us with the FcyRIIA CDNA and Dr. Susheela Tridandapani (Ohio State University College of Medicine, Columbus, OH, USA) for assistance in obtaining the FcyRIIA cDNA. We would also like to thank Mr. Akira Kotaki, Ms. Makiko Ikeda and Ms. Kazumi Yagasaki (National Institute of Infectious Diseases, Japan) for their excellent technical support. The authors are indebted to the participants of this study and the hospital and research institute staff for their technical support and contributions to this study.

\section{Funding}

This work was supported by funding from Japan Agency for Research and Development (AMED, H26-shinkou-jitsuyouka-007) and a grant-in-aid for Scientific Research (Wakate B no. 26870872) from the Ministry of Education, Culture, Sports, Science and Technology of Japan and funding from Nagasaki University. The funders had no role in the study design or the outcome of the study.

\section{Availability of data and materials}

All datasets on which the manuscript conclusions rely on has been presented in the main text and supplementary files. Detailed data sets and protocols and list of materials used (primers and probe sequences) are available upon request. Availability of materials are subject to institutional rules and regulations.

\section{Authors' contributions}

YS carried out the immunoassay, DENV infection assays, performed data analyses and drafted the manuscript. MLM carried out DENV infection assays, contributed reagents and materials, conceived the study, participated in study design and coordination, and drafted the manuscript. NT collected clinical samples, contributed reagents and data analyses. C-KL carried out immunoassay and JEV infection assay, contributed reagents and data analyses. $\mathrm{HJ}, \mathrm{KH}$ and MS participated in data interpretation and study design. IK and TT participated data interpretation, contributed reagents and materials and study design, and study coordination. All authors read and approved the final manuscript. 


\section{Competing interests}

The authors declare that they have no competing interests.

\section{Consent for publication}

Not applicable.

\section{Ethics approval and consent to participate}

Samples were collected at the National Center for Global Health and Medicine (NCGM), and all laboratory tests were performed at the National Institute of Infectious Diseases, Japan (NIID). The study protocols were approved by the Institutional Ethics Review Board of NCGM and NIID (no. 473). All samples were de-identified prior to tests conducted at the NIID.

\section{Author details}

'Department of Virology 1, National Institute of Infectious Diseases, Tokyo 162-8640, Japan. ${ }^{2}$ College of Bioresource Science, Nihon University, Fujisawa, Kanagawa 252-0880, Japan. ${ }^{3}$ Department of Virology, Institute of Tropical Medicine, Nagasaki University, Sakamoto 1-12-4, Nagasaki, Nagasaki 852-8523, Japan. ${ }^{4}$ National Center for Global Health and Medicine, Tokyo 162-8655, Japan. ${ }^{5}$ National Institute of Infectious Diseases, Tokyo 162-8640, Japan. ${ }^{6}$ Kanagawa Prefectural Institute of Public Health, Chigasaki, Kanagawa 253-0087, Japan

Received: 29 April 2016 Accepted: 27 September 2016

\section{Published online: 18 October 2016}

\section{References}

1. Bhatt S, Gething PW, Brady OJ, et al. The global distribution and burden of dengue. Nature. 2013;496:504-7.

2. Kutsuna S, Kato Y, Moi ML, Kotaki A, Ota M, Shinohara K, et al. Autochthonous dengue fever, Tokyo, Japan, 2014. Emerg Infect Dis. 2015;21(3):517-20.

3. Singh KP, Mishra G, Jain P, Pandey N, Nagar R, Gupta S, et al. Co-positivity of anti-dengue virus and anti-Japanese encephalitis virus IgM in endemic area: co-infection or cross reactivity? Asian Pac J Trop Med. 2014;7(2):124-9.

4. Pipattanaboon C, Sasaki T, Nishimura M, Setthapramote C, Pitaksajjakul P, Leaungwutiwong $P$, et al. Cross-reactivity of human monoclonal antibodies generated with peripheral blood lymphocytes from dengue patients with Japanese encephalitis virus. Biologics. 2013;7:175-87.

5. Mansfield KL, Horton DL, Johnson N, Li L, Barrett AD, Smith DJ, et al. Flavivirus-induced antibody cross-reactivity. J Gen Virol. 2011;92(Pt 12): 2821-9.

6. Calisher $\mathrm{CH}$, Karabatsos N, Dalrymple JM, Shope RE, Porterfield JS, Westaway EG, et al. Antigenic relationships between flaviviruses as determined by cross-neutralization tests with polyclonal antisera. J Gen Virol. 1989;70(Pt 1):37-43.

7. Kou Z, Quinn M, Chen H, Rodrigo WW, Rose RC, Schlesinger JJ, et al. Monocytes, but not T or B cells, are the principal target cells for dengue virus (DV) infection among human peripheral blood mononuclear cells. J Med Virol. 2008:80(1):134-46.

8. Littaua R, Kurane I, Ennis FA. Human IgG Fc receptor II mediates antibody-dependent enhancement of dengue virus infection. J Immunol. 1990;144(8):3183-6.

9. Tesh RB, Travassos da Rosa AP, Guzman H, Araujo TP, Xiao SY. Immunization with heterologous flaviviruses protective against fatal West Nile encephalitis. Emerg Infect Dis. 2002;8(3):245-51.

10. Bosco-Lauth A, Mason G, Bowen R. Pathogenesis of Japanese encephalitis virus infection in a golden hamster model and evaluation of flavivirus crossprotective immunity. Am J Trop Med Hyg. 2011;84(5):727-32.

11. Makino $Y$, Tadano M, Saito M, Maneekarn N, Sittisombut N, Sirisanthana V, et al. Studies on serological cross-reaction in sequential flavivirus infections. Microbiol Immunol. 1994;38(12):951-5.

12. Anderson KB, Gibbons RV, Thomas SJ, Rothman AL, Nisalak A, Berkelman RL, et al. Preexisting Japanese encephalitis virus neutralizing antibodies and increased symptomatic dengue illness in a school-based cohort in Thailand. PLoS Negl Trop Dis. 2011;5(10):e1311.

13. Halstead SB, Porterfield JS, O'Rourke EJ. Enhancement of dengue virus infection in monocytes by flavivirus antisera. Am J Trop Med Hyg. 1980; 29(4):638-42.
14. Li J, Gao N, Fan D, Chen H, Sheng Z, Fu S, Liang G, et al. Cross-protection induced by Japanese encephalitis vaccines against different genotypes of Dengue viruses in mice. Sci Rep. 2016;6:19953.

15. Kanesa-Thasan N, Putnak JR, Mangiafico JA, Saluzzo JE, Ludwig GV. Short report: absence of protective neutralizng antibodies to West Nile virus in subjects following vaccination with Japanese encephalitis or dengue vaccines. Am J Trop Med Hyg. 2002;66(2):115-6.

16. Posadas-Herrera G, Inoue S, Fuke I, Muraki Y, Mapua CA, Khan AH, et al. Development and evaluation of a formalin-inactivated West Nile Virus vaccine (WN-VAX) for a human vaccine candidate. Vaccine. 2010;28(50):7939-46.

17. Heinz FX, Stiasny K. Flaviviruses and flavivirus vaccines. Vaccine. 2012;30(29): 4301-6.

18. Coller BA, Clements DE. Dengue vaccines: progress and challenges. Curr Opin Immunol. 2011;23(3):391-8.

19. Ishikawa T, Yamanaka A, Konishi E. A review of successful flavivirus vaccines and the problems with those flaviviruses for which vaccines are not yet available. Vaccine. 2014;32(12):1326-37.

20. Takeshita N, Lim CK, Mizuno Y, Shimbo T, Kotaki A, Ujiie M, et al. Immunogenicity of single-dose Vero cell-derived Japanese encephalitis vaccine in Japanese adults. J Infect Chemother. 2014;20(4):238-42.

21. Moi ML, Lim CK, Kotaki A, Takasaki T, Kurane I. Detection of higher levels of dengue viremia using FcyR-expressing BHK-21 cells than FcyR-negative cells in secondary infection but not in primary infection. J Infect Dis. 2011; 203(10):1405-14.

22. Moi ML, Lim CK, Chua KB, Takasaki T, Kurane I. Dengue virus infectionenhancing activity in serum samples with neutralizing activity as determined by using FcyR-expressing cells. PLoS Negl Trop Dis. 2012;6(2):e1536.

23. Moi ML, Takasaki T, Saijo M, Kurane I. Dengue virus infection-enhancing activity of undiluted sera obtained from patients with secondary dengue virus infection. Trans R Soc Trop Med Hyg. 2013;107(1):51-8.

24. Frey A, Di Canzio J, Zurakowski D. A statistically defined endpoint titer determination method for immunoassays. J Immunol Methods. 1998; 221(1-2):35-41.

25. Konishi $E$, Tabuchi $Y$, Yamanaka A. A simple assay system for infectionenhancing and -neutralizing antibodies to dengue type 2 virus using layers of semi-adherent K562 cells. J Virol Methods. 2010;163(2):360-7.

26. Halstead SB, O'Rourke EJ. Dengue viruses and mononuclear phagocytes. I. Infection enhancement by non-neutralizing antibody. J Exp Med. 1977; 146(1):201-17.

27. Mady BJ, Erbe DV, Kurane I, Fanger MW, Ennis FA. Antibody-dependent enhancement of dengue virus infection mediated by bispecific antibodies against cell surface molecules other than Fc gamma receptors. J Immunol. 1991;147(9):3139-44.

28. Moi ML, Lim CK, Takasaki T, Kurane I. Involvement of the Fc gamma receptor IIA cytoplasmic domain in antibody-dependent enhancement of dengue virus infection. J Gen Virol. 2010;91(Pt 1):103-11.

29. Rodrigo WW, Jin X, Blackley SD, Rose RC, Schlesinger JJ. Differential enhancement of dengue virus immune complex infectivity mediated by signaling-competent and signaling-incompetent human Fcgamma RIA (CD64) or FcgammaRIIA (CD32). J Virol. 2006;80(20):10128-38.

30. Costin JM, Zaitseva E, Kahle KM, Nicholson CO, Rowe DK, Graham AS, et al. Mechanistic study of broadly neutralizing human monoclonal antibodies against dengue virus that target the fusion loop. J Virol. 2013;87(1):52-66.

31. Fibriansah G, Tan JL, Smith SA, de Alwis R, Ng TS, Kostyuchenko VA, et al. A highly potent human antibody neutralizes dengue virus serotype 3 by binding across three surface proteins. Nat Commun. 2015;6:6341.

32. Kliks SC, Nisalak A, Brandt WE, Wahl L, Burke DS. Antibody-dependent enhancement of dengue virus growth in human monocytes as a risk factor for dengue hemorrhagic fever. Am J Trop Med Hyg. 1989;40(4):444-51.

33. Libraty DH, Endy TP, Houng HS, Green S, Kalayanarooj S, Suntayakorn S, Chansiriwongs $W$, et al. Differing influences of virus burden and immune activation on disease severity in secondary dengue-3 virus infections. J Infect Dis. 2002;185(9):1213-21.

34. Singla M, Kar M, Sethi T, Kabra SK, Lodha R, Chandele A, et al. Immune response to dengue virus infection in pediatric patients in New Delhi, India-Association of Viremia, Inflammatory Mediators and Monocytes with Disease Severity. PLoS Negl Trop Dis. 2016;10(3):e0004497.

35. Hoke CH, Nisalak A, Sangawhipa N, Jatanasen S, Laorakapongse T, Innis BL, et al. Protection against Japanese encephalitis by inactivated vaccines. N Engl J Med. 1988;319(10):608-14. 
36. Sato R, Hamada N, Kashiwagi T, Imamura Y, Hara K, Naito Y, et al. Dengue hemorrhagic fever in a Japanese traveler who had preexisting Japanese encephalitis virus antibody. Trop Med Health. 2015;2:85-8.

37. Izurieta RO, Macaluso M, Watts DM, Tesh RB, Guerra B, Cruz LM, et al. Anamnestic immune response to dengue and decreased severity of yellow fever. J Glob Infect Dis. 2009; 1 (2):111-6.

38. Lim CK, Takasaki T, Kotaki A, Kurane I. Vero cell-derived inactivated West Nile $(W N)$ vaccine induces protective immunity against lethal WN virus infection in mice and shows a facilitated neutralizing antibody response in mice previously immunized with Japanese encephalitis vaccine. Virology. 2008; 374(1):60-70.

39. Libraty DH, Nisalak A, Endy TP, Suntayakorn S, Vaughn DW, Innis BL. Clinical and immunological risk factors for severe disease in Japanese encephalitis. Trans R Soc Trop Med Hyg. 2002;96(2):173-8.

Submit your next manuscript to BioMed Central and we will help you at every step:

- We accept pre-submission inquiries

- Our selector tool helps you to find the most relevant journal

- We provide round the clock customer support

- Convenient online submission

- Thorough peer review

- Inclusion in PubMed and all major indexing services

- Maximum visibility for your research

Submit your manuscript at www.biomedcentral.com/submit
Biomed Central 